\title{
Dancing with DNA and flirting with the ghost of Lamarck
}

\author{
MARY JANE WEST-EBERHARD \\ Smithsonian Tropical Research Institute, c/o Escuela de Biología, Cìdad Universitaria, Costa Rica \\ (e-mail:mjwe@sent.com; phone: +506-228-0001; fax: +506-228-0001)
}

Review of: Evolution in Four Dimensions, Eva Jablonka and Marion J. Lamb, 2005, MIT Press, Cambridge, Massachusetts

Evolution in Four Dimensions, by Eva Jablonka and Marion Lamb, is a disarmingly good-humored book that challenges the overly gene-centered 'NeoDarwinian' (mid-20th-Century-Synthesis) view of evolution via selection on phenotypes affected by random changes in DNA. Their remedy, more palatable by the end of the book than I expected at the beginning, is to propose that we revise and expand our "unidimensional' vision of heredity. Heredity, they argue, occurs in four dimensions: genetic inheritance, the conventionally recognized mode of inheritance via transmission of DNA; epigenetic inheritance, or transmission of non-genetic information from parental cells to daughter cells, as in the cytoplasm of an egg; behavioral inheritance, or cultural transmission of learned traits; and symbolic transmission of information by means of abstract representation, especially language in humans. All four modes of inheritance provide variants on which natural selection can act. Therefore they portray evolution as occurring in four dimensions, corresponding to the four dimensions of inheritance.

The style of the book makes it accessible to an educated lay reader. The text has been unburdened by removal of bibliographic citations to a single section of notes at the end of the book, and by a series of dialogues with a make-believe Devil's advocate named Ifcha Mistabra (in Aramaic, "The opposite conjecture"), where the authors anticipate and answer potential confusions and objections to their main points. All of the figures are in the form of whimsical drawings by Anna Zeligowski, whose illustrations manage to combine scientific accuracy with playful fantasy (on the book jacket she portrays the sober-faced Ifcha Mistabra dancing with a graceful double helix in high heels, while some friendly doctors attempt to decipher her code; Figure 1). These devices, and the very clear style of the writing, make for a delightfully readable book. There is an extensive bibliography, but I could not always find the references corresponding to findings that were new to me because the endnotes are not tagged by numbers or superscripts to statements in the text. And the dialogues did not always address the questions I would have raised. So I am grateful for this opportunity to continue the dialogue between the authors and their congenial challenger, Ifcha Mistabra. 


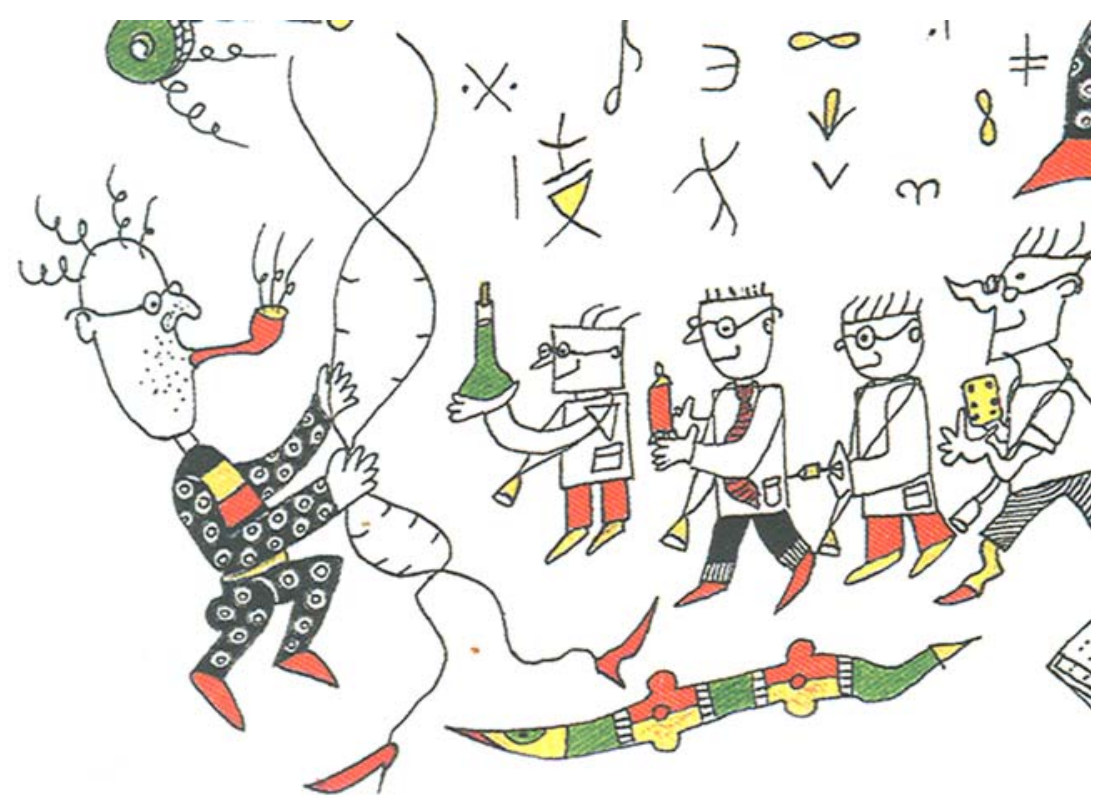

Figure 1. Ifcha Mistabra, DNA, and scientists. From the cover illustration of Evolution in Four Dimensions. Drawing by Anna Zeligowski.

In that spirit I would pursue one of Ifcha-Mistabra's own points and query whether the authors may have decided on the wrong approach to the right problem. They correctly identify an important shortcoming of mainstream evolutionary thought, namely, the neglect of environmentally induced variation and its importance for evolution by natural selection (all of their three added dimensions of inheritance involve the inheritance of environmentally induced variation). I did not find any of the claims made in this book to be fundamentally wrong. But by focusing on a solution in terms of inheritance I believe that the authors have argued themselves, unnecessarily, into a corner where they are obliged to defend Neo-Lamarckism if they are to reform 'NeoDarwinism:' by their line of reasoning, the inheritance of acquired characteristics must be acknowledged as a key concept if environmental influence on development is to be incorporated into evolutionary theory.

The book begins with a prologue that announces (p. 1) the coming of "a revolutionary change" in biological thinking about heredity and evolution. It lists four claims implied by that revolution: (a) there is more to heredity than genes; (b) some hereditary variations are nonrandom in origin; (c) some acquired information is inherited; and (d) evolutionary change can result from instruction as well as selection. This sounds revolutionary in the context of a strictly genetic concept of inheritance, but all of these heretical-sounding claims are acceptable if one simply remembers that there is cultural transmission of behavior patterns and agrees to call that a mode of 'inheritance.' Then it is 
clear that there is more to heredity than genes (all normal humans inherit behavior patterns taught by their parents); that some culturally transmitted variations are non-random or purposefully functional in origin (e.g., human inventions, or the much publicized food-washing traditions in Japanese monkeys); that such acquired (e.g., learned) information is inherited, in the sense of being passed from one generation to the next; and that (cultural) evolution (cross-generational change in behavior) can result from instruction as well as selection.

In the final chapter of the book (pp. 355-356) Ifcha Mistabra makes this same point: "I doubt that anyone will dispute..." the first three claims of the Prologue, he says; and the fourth, regarding behavioral evolution, is acceptable if evolution is defined as cross-generational phenotypic change that need not involve genetic change. The thrust of the book, then, is a call to attention, not a sweeping reform: non-genetic inheritance is familiar to all of us. What forms does it take, and what might this mean for evolution? Ultimately, that is the message of the book. Part of its style is to have initially provocative statements softened or qualified later on, a game of provoke-and-mollify that may have been inspired by playing with the cat mentioned in the acknowledgements.

The announcement of a revolution concerning heredity effectively piques the interest of a reader, but it is a revolution without a cause if its key claims are acceptable without a change of mind. While this comment concerns, in part, a tactic of presentation it also concerns substance, because it suggests that a narrow view of inheritance may not be the primary shortcoming of current evolutionary thought - that the heart of the problem may lie elsewhere. This book convinced me that an expanded view of inheritance contributes to the solution of a more pervasive problem - the neglect of environmental factors in development and of development in evolution. Since development (including learning and other epigenetic phenomena) is the source of all selectable phenotypic variation, environmentally influenced development, and (when it occurs) the inheritance of developmental variants, affect evolution.

In one respect focusing on environmentally influenced inheritance truly is more revolutionary than focusing on environmentally influenced development. Environmental effects on development have long been largely ignored in evolutionary biology, but they have at least been given lip service, whereas the inheritance of acquired traits has been both ignored and abjured. The ban against Lamarckism has meant a reflex denial that the inheritance of acquired traits, in any form, can occur. So the number of examples unearthed in this book will surprise many readers, as will the reminder that cultural transmission is a kind of inheritance of acquired traits. The first, most controversial place that Jablonka and Lamb look for environmental effects on inheritance is in the genome itself. They begin with an admirably clear explanation of the structure and behavior of DNA and RNA, and of the role of the intracellular environment in transcription and gene expression. They describe several ways in which the genome is modified and manipulated during development, including contributions of alternative splicing, polyploidy, amplification of expression 
via polytene chromosome formation and duplication, and deletions. And they include a good critique of "genetic astrology" among scientists and in the popular press, where complex human traits such as homosexuality shyness, and religiosity are attributed to particular genes. In the chapter titled "From genes to phenotype" the environmentally induced genomic changes described occur in the somatic, not the transmitted genome, so they are not inherited genomic changes that would affect evolution by any means so far known. I sometimes wished that the text had been clearer about the importance of this distinction between the somatic and the transmitted genome. Jablonka and Lamb leave that crucial distinction to the very last sentence of the dialogue at the end of this chapter, where they indicate that the relevance to cross-generational inheritance remains to be seen: "We therefore need to study the systems of nonrandom DNA change [in response to environmental cues] and what kind of heritable genetic variation is formed through them, and whether they influence genetic variation in the next generation. If they do, it changes the way we have to think about the role of the environment in evolution" (p. 78) (italics mine).

Jablonka and Lamb describe DNA as containing "information" and gene action as involving "interpretation." They explain that "information" is recognized if there is a source and a "receiver that reacts to this source and interprets it," meaning that "...the receiver's functional state is changed in a way that is related to the form and organization of the source" (p. 53). It does not seem necessary to introduce the concept of 'interpretation' into the discussion of gene action. As in the study of animal communication, it is preferable not to appear to attribute special abilities to organisms but to describe what is actually observed, here, that a particular input or stimulus consistently evokes a particular response, with the response distinctive for different receivers and consistently correlated with the form of the input. If I throw a brick into sand I get one type of response, and if I throw water into sand I get another. It does not add to the understanding of this difference to say that it involves 'interpretation,' and it may prove easier to analyze the actual mechanisms of phenotypic responses to environmental and genomic inputs if we leave the notion of interpretation aside.

Moving on to a possible role of environmental change in the induction of genomic change (mutation), they make the important point that "It would be very strange indeed to believe that everything in the living world is the product of evolution except one thing - the process of generating [genetic] variation!" (p. 101). This is not a completely neglected area in evolutionary biology. Quite early in the Synthesis era theoretical geneticists (references in Leigh 1973) considered the adaptive evolution of mutation rates, and they discussed early studies of bacteria that revealed mutation 'hot spots' (Benzer 1957) and experimental alteration of mutability (Zamenhof et al. 1966). Jablonka and Lamb cite some recent theory of this sort. They take a new look at the subject of directed mutation and find limited evidence for what they term "interpretive mutations," which "fall at different points on the spectrum between totally random and totally directed mutation" (p. 43). They describe examples of 
stress-mediated "induced global mutations" (increased mutation rate that is not specific to particular loci); "local hypermutation" (zone-specific mutational hot spots in DNA); and induced local mutations affecting particular genes. Again, labeling genomic responses (mutation) as 'interpretive' seems unnecessary. But the discussion of induced mutation, primarily in bacteria, is thought-provoking because Jablonka and Lamb focus hard on the possible evolutionary significance of these relatively neglected phenomena, especially their role in the early evolution of life. Their ruminations, in later chapters, regarding the ancient origins of the developmental mechanisms observed in metazoan somatic cells are particularly interesting.

"Epigenetic" inheritance refers to non-DNA transmission of information from parent to descendent cells. Chapter 4, on epigenetic inheritance systems, endeavors to show that non-genetic cellular inheritance systems are important in evolution. It uses a thought experiment to show "that it is possible to think about evolutionary change based solely on variations transmitted by non-genetic cellular inheritance systems" (p. 114) (italics mine). It then describes four broad categories of epigenetic inheritance whose "evolutionary potential"(again, italics mine) has been overlooked: self-sustaining feedback loops that maintain particular patterns of gene-expression in lines of differentiated cells; structural inheritance based on use of parental-cell architecture as a template for daughter-cell structure, as in the ciliary rows of ciliates, and in the reproduction of prions; chromatin marking systems, as in DNA compaction using histone groups, and DNA methylation which blocks transcription of particular methylated chromosome regions during development; and RNA interference, which leads to the silencing of particular genes. The authors provide exceptionally clear descriptions of how these processes survive chromosome duplication during multiplication of somatic cells.

Three of the four epigenetic processes discussed occur in somatic cells during individual development, the one exception being templating in single-cell eukaryotes and prions. How important are these three somatic-cell processes in evolutionary change, which requires cross-generational inheritance, not just inheritance in somatic cell lineages? The answer to this question occupies the final section of the chapter, which concludes that cross-generational transmission can occur by all four mechanisms in single-celled eukaryotes; that methylation marks can be transmitted in bacteria and (in a few cases) mammals and plants showing parental "genomic imprinting," a phenomenon whose cross-generational transmission is transient, however, because the marks are erased, as the authors point out, when the chromosome passes from one sex to the other. They cite two examples where transmission of a chromosomal mark is stable across many generations, a methylation that affects flower form in the plant toadflax (Linaria vulgaris), and an unusual yellow fur color and associated traits discovered in a mutant laboratory strain of mice, due to a methylated transposon that is maternally transmitted via the egg. Since transposon sequences are extremely common in the mammalian genome this mode of transmission of non-genetic effects may be more common than currently 
realized. They conclude that "evolution can occur through the epigenetic dimension of heredity even if nothing is happening in the genetic dimension" (p. 144). In short, epigenetic evolution is feasible. The authors argue that may be common. At the very least, the evidence demands keeping an open mind.

The discussion of epigenetic inheritance, like the chapter on genomic change, might have been improved by starting with greater clarity regarding the crucial distinction between somatic and cross-generational inheritance. In order for "epigenetic" effects (environmental effects on the genome or on cells) to be important in evolution they have to be passed between generations. Single-celled organisms can transmit environmentally induced alternations across generations, and the authors effectively argue that this may be important in bacteria and paramecia, and may have characterized inheritance in primitive organisms soon after the origin of life. The possibility of inherited stress-related genetic variation mediated by the activity of transposable elements, as in McClintock's studies of maize, also is arguably important in evolution plants and mammals. But for epigenetic inheritance to be of importance for evolution in metazoans, the germline cells, or other undifferentiated cells that are the starting points for a new generation, have to be affected. Such undifferentiated cells are not subjected to the full array of environmentally sensitive processes of development and differentiation that affect somatic cells. So the authors' many examples of non-genetic inheritance across generations of differentiated cells, while of great interest for understanding development, and nicely explained in this book, cannot be extrapolated to cross-generational inheritance between individuals. Somatic cell lines die with the individual regardless of their reproductive success within the individual. Jablonka and Lamb acknowledge this near the end of the chapter when they write "It is when we think about the transmission of epigenetic variants through sexual generations that theoretical difficulties arise" (p. 138).

The difficulties are more than theoretical. There are numerous mechanisms in addition to germline sequestration, in a variety of multicellular and syncitial organisms, that prevent cross-generational transmission of epigenetic effects. Such mechanisms and the epigenetic effects whose cross-generational transmission they curb are discussed for a wide variety of types of organisms in Buss (1986), The Evolution of Individuality, a book that could serve as a cautionary companion volume to the discussion of epigenetic inheritance in Evolution in Four Dimensions. Like Jablonka and Lamb, Buss argues that epigenetic effects have been neglected by evolutionary biologists. But he also shows why to expect powerful selection against cross-generational epigenetic inheritance because of the importance of individual integrity under selection, and its predominance over sub-individual (genic or cell-lineage) selection. ${ }^{1}$ To their credit,

\footnotetext{
${ }^{1}$ Readers need to be aware that Buss's hypothesis regarding the origin of cell-lineage differentiation in metazoans is flawed because it argues that such differentiation must originate due to competitive interactions among differentiated cell types prior to the sequestration of the germline. The origin and selection of developmental pathway divergence need not be restricted in this way. For references and discussion see Raff (1988) and others cited by Jablonka and Lamb (1995) and WestEberhard (2003).
} 
Jablonka and Lamb include a Buss-like discussion of this problem in Chapter 7. They then give examples sufficient to convince that a rigid denial of the feasibility of epigenetic inheritance is unjustified, especially in an age when the technical difficulties of demonstrating such phenomena are only beginning to be overcome. But these examples are as yet too few to be completely convincing about the general importance of cross-generational epigenetic inheritance for evolution.

The chapter on behavioral inheritance is more convincing. It illustrates two types of behavioral transmission, those based on transfer of behavior-influencing substances from mother to offspring, which could be termed maternal effects on the behavioral phenotype; and transmission via learning. As examples of transmission via substances they describe the preferences of rabbit pups and human babies for foods eaten by their mothers during pregnancy, during lactation, and, in rabbits, by ingestion of maternal feces. Transmission via learning occurs in such phenomena as 'imprinting' during a critical period, which is known to produce local evolutionary change in such traits as male song and female preference for parental song in birds; observational learning about new food sources with local cultural spread of their exploitation, as in the famous robbing of milk from bottles by various species of birds in Europe; and cultural evolution in Japanese macaques that across many generations has produced complex behavioral changes shared by many individuals in a population.

It is not clear that behavioral inheritance alone would very often lead to trait persistence across generations. Unlike genetic inheritance, where the inherited elements of a capacity to produce a behavioral or morphological phenotype can be transmitted even if the phenotype is not expressed, a culturally transmitted trait like spoken language or unwritten music can be completely lost in a single generation if not expressed and learned. Similarly, a maternally influenced trait like diet may not be consistent across life stages, environments, seasons and generations in nature. In several places the authors mention the inheritance of dietary preferences due to larval conditioning via ingestion of the larval food plant chosen by the mother, citing Darwin as discussing this in cabbage butterflies. This hypothesized mechanism, known as the "Hopkins host-selection principle," has been extensively investigated by entomologists but not to my knowledge shown to work (this is a place where I wished for a supporting reference in the text). The chain of behavioral inheritance would end if other factors, such as host-plant scarcity, adaptive diet diversification (the ingestion of a variety of essential foods), or individual differences in experience and foraging abilities, intervene to affect maternal diet. Nonetheless, such behavioral transmission, even if lasting only a few generations, could speed the evolution of specialization via genetic evolution when favored by selection, as the authors argue in a later chapter. So the primary evolutionary contribution of behavioral inheritance via maternal effects may be to bias genetic evolution, including genetic accommodation of learning as a mechanism that contributes to specialized preference. Even though relatively 
susceptible to loss when unexpressed, behavioral imprinting, observational learning, and cultural transmission represent undoubted examples of crossgenerational behavioral evolution that need not involve genetic change.

The book makes the important point that "instability" of behavior patterns is more likely to prove adaptive than variation arising in the genome: genetic variants (mutations) are likely to be detrimental to function, but learned behavioral variants are more likely to be adaptive, since they are filtered by an evolved learning process (involving, for example, context-specific motivation and reinforcement) that makes them more likely to maintain function, or even create new function, during change. Jablonka and Lamb call this adaptedness the "educated guess" property of behavioral variation. Although they do not mention this, the same point regarding maintenance of function is true to some extent for non-learned phenotypic variants, including those induced by mutation. Traits induced by mutation and environmental factors are similarly filtered, being produced during development by a pre-existing, responsive phenotypic structure that has evolved under natural selection and can adaptively accommodate change, even if not able to invent adaptive novelties the way an intelligent vertebrate can.

In this and other places I thought that the strong focus on inherited variation actually limited the authors' ability to apply their insights more broadly, to environmentally influenced development in general, in order to show the broader significance of environmentally induced phenotypes in evolution. Beginning with reader skepticism regarding the inheritance of acquired traits, they have an uphill battle to show the importance of environmental factors beginning with inheritance. Readers will keep wondering how important these alternative modes of inheritance are, compared to conventional genetics. Are they important enough to lead a revolution in evolutionary biology?

On this, the "comparative question," Ifcha Mistabra seemed overly kindhearted, and I sometimes suspected him of being in cahoots with the authors. He acquiesced far too easily when, in response to his question about the relative importance of different modes of inheritance, they responded: "in a sense that is an absurd question" (p. 362). In one sense the 'comparative question' truly is absurd, for all modes of inheritance are of interest for a general theory of phenotypic evolution. But in another sense, it is not so absurd to expect a key element of a proposal for sweeping reform of evolutionary theory to be based on common, not unusual, phenomena.

Learning, for example, is common among animals, but cross-generational transmission of learned traits is not so common. What, then, is the evolutionary significance of learning? Contrary to common belief, learned variants are especially subject to genetic modification under selection because of their capacity for consistent environment matching and local recurrence, within and sometimes between generations. The mechanisms and morphologies of behavior are as subject to genetic variation as any other traits, and so the frequency (spread) and form of such variants will inevitably evolve as a response to selection via conventional genetic means. Given that reinforcement 
learning is extremely widespread in animals, including in unicellular species and others that lack cross-generational transmission of learned traits, the evolutionary effect of learning for the origins of novelty is probably great, and its evolutionary effect is probably usually via genetic rather than via nongenetic inheritance.

As Jablonka and Lamb discuss, symbolically transmitted traits could have genetic evolutionary effects to the degree that their variations influence group or individual reproductive success. The extent to which symbolic evolution may take on a life of its own, independent of any possibility of genetic modification, is an area of vigorous debate and few data, where I prefer not to tread. But it is safe to say that this is a kind of transmission seen in only one or a few species. And, as already discussed, cross-generational epigenetic inheritance is largely blocked by devices that have evidently evolved to prevent it.

It is difficult to avoid the conclusion that the three alternative modes of inheritance - epigenetic, behavioral/cultural, and symbolic - are somewhat special cases, or at least not yet known to be common enough among organisms to be of broad general importance for evolution. And since all expressed traits, inherited or not, may be subject to genetic variation and selection, I conclude that conventional genetic inheritance is likely to be by far the most important mode of transmission for evolution. This is not to say that the three alternative modes of inheritance discussed by Jablonka and Lambare unimportant for evolution, but only to say that their emphasis on phenomena that may be seen by most readers as relatively rare may not be the best way to start a revolution.

This conclusion does not deny the need for a case-by-case evaluation - one reason Jablonka and Lamb considered 'the comparative question' of relative importance compared to genetic inheritance "in a sense absurd." Their other reason - that it is like asking which is more important, genes or environment in development - is not a valid objection, as a more rebellious and persistant Ifcha Mistabra might have pointed out. The dual genetic-environmental causation of development is widely acknowledged to be a universal property of development, and the intertwined effects of the two influences have been passed between generations to varying degrees since the origin of phenotype reproduction itself. So questioning which is more important truly is absurd. Non-genetic inheritance, by contrast, is not a universal property of phenotypic traits, and its importance is not universally acknowledged; so it is not comparably absurd to ask how important it is relative to genetic inheritance as a factor in phenotypic evolution.

The emphasis in this book on inherited units of variation, rather than on a more inclusive concept of environmentally influenced variation, is associated with a particular view about the units of selection. In early chapters Jablonka and Lamb treat the units of inheritance as the units of selection. In the Prologue (p. 1) they state that "the usual version of Darwin's theory of evolution...is that adaptation occurs through natural selection of chance genetic variations." Their critique of this version focuses, not on the gene-selection 
aspect, which at this point they accept or overlook. Rather, they criticize the idea that the selected variations must be genetically inherited to be important for evolution, and they proceed to discuss other modes of inheritance, which "also provide variation on which natural selection can act" (p. 1). "By adopting a four-dimensional perspective, it is possible to construct a far richer and more sophisticated theory of evolution, where the gene is not the sole focus of natural selection" (p. 2). The implication here seems to be that, in their conception of evolution, selection acts on the units of inheritance - genes, and other inherited entities (epigenetic, behavioral, symbolic). On p. 41 they refer to "units of heredity and selection,", again implying that the units of heredity are the units of selection.

Later in the book the phenotype is affirmed to be the unit of selection, though it was not always clear whether that includes only inherited phenotypes that are transmitted across generations by epigenetic, behavioral or symbolic means. In the discussion of genetic assimilation (Chapter 7), "selectable phenotypes" are described (p. 262). That chapter, on the interactions between genes and epigenetic systems, discusses how "...an induced epigenetic change [in the phenotype] that occurs repeatedly can guide the selection of genes that produce the same phenotype." In that sentence selection on genes is guided by a (changed) phenotype: selection would seem to be on both phenotypes and on genes. To be consistent with selection on phenotypes, that sentence should read "an induced epigenetic change...can guide selection [on phenotypes] that favors genes that bias development toward production of the same phenotype." That is, selection acts on phenotypes and only secondarily changes the frequencies of underlying genes.

Jablonka and Lamb thus appeared to modify their concept of selection when they got to the discussion of genetic assimilation. At the outset, the units of selection were the units of inheritance, which would seem to include genes; later, genes are excluded. They regard selection as being on phenotypes, but specify that they mean 'heritable' phenotypes (meaning, presumably those that are passed between generations), and not genes: “...we prefer to think about the selection of heritable phenotype units, rather than genes" (p. 280). In conventional Synthesis terms, selection on the genetically influenced phenotype, not inheritance of acquired traits, is what allows genetic assimilation to work, and their description of the inherited basis of evolution by genetic assimilation is exactly as described under the Synthesis: "Short-term evolution [involving genetic assimilation] does not depend on new mutations, but it does depend on epigenetic changes that unveil the genetic variants already present in the population” (p. 275) (cf. Mayr 1963, p. 190: “...the essential point [about genetic assimilation is that it] reveals which among a number of individuals already carry polygenes or modifiers of the desired phenotype."

The attempt to discern how the authors define the units of selection in relation to the units of inheritance is not merely pedantic. The structure and consistency of a theory of adaptive evolution is revealed by how the word 'selection' is used in relation to 'phenotype,' 'evolution,' and the units of 
inheritance (e.g. genes, gemmules, or phenotypic entities passed between generations). The Darwinian theory of evolution clearly specifies the nature of cause and effect involving these terms, contrary to claims that it is tautological: differential survival and reproduction ( $\equiv$ selection) depends upon phenotypic differences between the reproducing entities ( $\equiv$ the units of selection); and if those phenotypic and reproductive-success differences are correlated with differences in inherited entities (genes $\equiv$ the units of inheritance), the frequencies of the inherited entities change from generation to generation, causing phenotypic change accompanied (in modern terms) by gene-frequency change ( $\equiv$ evolution). I wish Ifcha Mistabra had asked for a comparably well-defined scheme of cause-and-effect to fit each of the four dimensions of evolution visualized by Jablonka and Lamb. When an inherited epigenetic trait is the unit of selection, what determines its frequency in the next generation? Is it simply self-perpetuated by the various mechanisms of non-genetic inheritance they describe, as long as the cell divides? And if so, doesn't that self-perpetuation depend on the (evolved, genetically influenced) mechanisms, that can be either maintained or eliminated under selection on a higher-level entity (the cell, or the individual)? This would mean that the frequency of the trait ultimately depends upon conventional selection and genetics. The same could be said for learned, culturally transmitted traits: they can be perpetuated by learning, but that perpetuation depends on the maintenance and the precise nature of the evolved ability to learn. Perhaps the best prospect for non-genetic evolution independent of genetically mediated evolution is in the realm of symbolic inheritance; but even there, Jablonka and Lamb discuss the many possible interactions between symbolic systems and the evolved, genetically influenced ability to symbol. Is it ever reasonable to consider epigenetic inheritance and epigenetic evolution in complete isolation from the evolution of a higher unit (the individual, or the social group) which is the unit of reproduction? I think the answer is no: the four dimensions of inheritance do not correspond to four truly distinct dimensions of evolution. Rather, as Jablonka and Lamb end up discussing when they get down to the business of "Putting Humpty-Dumpty together again" (Part III), higher units of selection and gene-frequency change end up getting involved.

It is important to note that Darwinian adaptive evolution, as formulated by Darwin, is phenotypic change accompanied by change in the material of inheritance (today: gene-frequency change), not gene-frequency change alone, although sometimes erroneously caricatured as such. It is now clear that genetic evolution, e.g. of so-called 'neutral' alleles, can occur without selection, via drift, but that process is independent of selection on phenotypes and so is not Darwinian (adaptive) evolution. The observations of Jablonka and Lamb challenge us to re-examine the Darwinian framework, and their discussion does show weaknesses in the Darwinian approach. For example, if our primary focus is phenotypic change accompanied by gene-frequency change, what do we do about phenotype frequencies that can change across generations due to the transmission of the phenotypes themselves, without genetic change? Such 
purely phenotypic evolution could even be quasi-Darwinian in that it could involve selection (differential reproductive success of phenotypically different entities) without being genetic, if differential reproductive success affects the differential transmission of phenotypic variants uncorrelated with genetic variation. And it could be clearly 'Non-Darwinian' if it did not involve differential survival or reproductive success associated with different phenotypes, but depended entirely upon differential environmental induction (e.g., due to environmental change). This latter type of phenotypic evolution may be fairly common in nature, for example when one of a pair of alternative phenotypes in a polyphenism is locally fixed or otherwise changed in frequency within a population due to change in local environmental conditions. Within the formalism of standard Darwinian theory, such change would not be regarded as 'evolutionary' change and phenotype frequencies might readily and repeatedly change, due to temporal or spatial environmental change.

This distinction, under conventional theory, between 'evolutionary' and 'non-evolutionary' phenotypic change begins to split hairs. But the distinction is perhaps useful, if only to keep the mechanisms of change straight. The important thing is not to derisively dismiss "non-evolutionary" or "Lamarckian" change of the sorts discussed by Jablonka and Lamb as somehow uninteresting for evolution. Evolutionary biology needs to examine the full gamut of possible mechanisms of cross-generational change, not least because any change in phenotype frequencies is highly likely to become important for evolution in the conventional sense of eventually involving a change in gene frequencies.

Along with Ifcha Mistabra I wondered why the authors insisted on identifying their ideas with Lamarck's: "If what you want to do is convince people ...why do you insist on using a term that, as you obviously recognize, makes people think that you are a pair of confused muddle-headed idiots?... Why not avoid 'Lamarckism' altogether...?" (p. 361). Part of their defense is their interest in history, and their wish to justly acknowledge their intellectual debt to Lamarck, who, like Darwin, was neither completely correct, nor completely wrong. But a study of history also shows that reference to Lamarck, for example, in Waddington's essays on the "genetic assimilation of an acquired trait" (1953), arouses objections more effectively than it courts acceptance. Skepticism toward Lamarckism persists, even though the authors believe that it should not ("it is time for people to be more open-minded about Lamarckism;" p. 362). I worry that linking environmentally mediated variation to specializedlooking alternative modes of non-genetic inheritance and thereby to Lamarckian processes may distract readers from the larger importance of environmental induction for evolution.

In sum, Evolution in Four Dimensions describes modern findings and fascinating examples showing that long-held beliefs about inheritance deserve reconsideration. In that respect, the book merits attention by all biologists. With its agreeable style it will painlessly raise their level of sophistication regarding different modes of inheritance, and the potential importance of 
non-genetic inheritance for evolution and during the history of life. In this book, the reader has the impression of being in the company of an amiable pair of rebels who rightfully stir up a complacent public. They cajole and educate us into being more broad-minded about the basic tenets of heredity and evolution. But the revolution in evolutionary biology, if it is to occur, will concern not so much the mechanisms of heredity - in fact, one of the strongest points of the earlier Synthesis - but the environmental causes of developmental variation of all sorts, not just inherited variation; and it will focus on the relation of that variation to modern genetics, as research on gene expression and associated genomic processes (like methylation, chromosomal condensation, alternative splicing and environmentally modulated mutation rates) converges with research on development and the phenotype, including the behavioral phenotype. Jablonka and Lamb have written an erudite and engaging book that will appeal to anyone who enjoys informed criticism, and who is interested in forward-looking ideas about heredity, development, and evolution.

\section{References}

Benzer S. 1957. The elementary units of heredity. In: McElroy W.D. and Glass B. (eds), The Chemical Basis of Heredity, Johns Hopkins University Press, Baltimore, pp. 70-93.

Buss L.W. 1987. The Evolution of Individuality. Princeton University Press, Princeton.

Jablonka E. and Lamb M.J. 1995. Epigenetic Inheritance and Evolution. Oxford University Press, New York.

Leigh E.G. Jr. 1973. The evolution of mutation rates. Gent. Suppl. 73: 1-18.

Mayr E. 1963. Animal Species and Evolution. Belknap Press, Cambridge, Massachusetts.

Raff R.A. 1988. The selfish cell lineage. BioEssays 14: 211-218.

Waddington C.H. 1953. Genetic assimilation of an acquired character. Evolution 7: 118-126.

West-Eberhard M.J. 2003. Developmental Plasticity and Evolution. Oxford University Press, New York.

Zamenhof S., Heldenmuth L.H. and Zamenhof P.J. 1966. Studies on mechanisms for the maintenance of constant mutability: mutability and the resistance to mutagens. Proc. Natl. Acad. Sci. USA 55: $50-58$. 